\title{
Uma Proposta Baseada em Internet das Coisas para Prover Acessibilidade a Pessoas com Deficiência Visual ${ }^{*}$
}

\author{
Cleiton Garcia ${ }^{1}$, Felipe Haertel ${ }^{1}$, Patricia Davet ${ }^{2}$, Gerson Cavalheiro ${ }^{2}$, \\ João Lopes ${ }^{3}$, Adenauer Yamin ${ }^{1}$ \\ ${ }^{1}$ Universidade Católica de Pelotas (UCPEL) - Pelotas - RS - Brasil \\ ${ }^{2}$ Universidade Federal de Pelotas (UFPEL) - Pelotas - RS - Brasil \\ ${ }^{3}$ Instituto Federal Sul-rio-grandense (IFSUL) - Pelotas - RS - Brasil \\ \{cleiton.garcia, felipe.haertel, adenauer.yamin\}@ucpel.edu.br, \\ \{ptdavet, gerson.cavalheiro\}einf.ufpel.edu.br \\ joaolopes@cavg.ifsul.edu.br
}

\begin{abstract}
The design of mobile and ubiquitous applications to serve people with visual impairment can be enabled due to the wide variety of available mobile devices, of different types, capabilities and technologies, associated with the profusion of wireless communication networks. In this sense, the main contribution of this paper is the approach, called HELIX, proposed to provide accessibility to the visually impaired, through the use of concepts of Internet of Things (IoT) in indoor and outdoor environments. The functional architecture of the HELIX is integrated into the adaptation and context recognition subsystem of EXEHDA middleware, which provides support for the acquisition, storage and processing of context information used by HELIX mobile applications. The applications prototyped were evaluated by the Technology Acceptance Model, and the results obtained point to the feasibility of providing accessibility to people with visual impairment through an approach based on Internet to Things.
\end{abstract}

Resumo. Com a ampla diversidade de dispositivos móveis disponíveis, de diferentes tipos, capacidades e tecnologias, associando-se à profusão das redes de comunicação sem fio, possibilita-se a oferta de serviços móveis e ubíquos que podem ser destinados a atender pessoas com deficiência visual (PCDVs). Nesse sentido, este artigo tem como contribuição central a proposta de uma abordagem, denominada HELIX, para prover acessibilidade às pessoas com deficiência visual através do emprego de conceitos da Internet das Coisas (Internet of Things - IoT) em ambientes Indoor e Outdoor. A arquitetura funcional da abordagem HELIX está integrada ao Subsistema de Reconhecimento de Contexto e Adaptação do middleware EXEHDA, o qual provê suporte para a aquisição, armazenamento e processamento das informações de contexto utilizadas pelas aplicações móveis da HELIX. As aplicações prototipadas foram avaliadas através do modelo TAM (Technology Acceptance Model), sendo que os resultados obtidos apontaram para a viabilidade de prover acessibilidade às pessoas com deficiência visual por meio de uma abordagem baseada na Internet das Coisas.

*Trabalho realizado com apoio da CAPES (Programa Nacional de Cooperação Acadêmica- Procad) e da FAPERGS (Programa Pesquisador Gaúcho - PqG) 


\section{Introdução}

As pessoas com redução ou perda da capacidade para a realização de tarefas visuais como ler, reconhecer objetos e rostos enfrentam inúmeras dificuldades no dia-a-dia devido a falta de recursos para acessibilidade, necessitando de suporte para que possam efetivamente exercer a cidadania plena [Silva et al. 2010].

Considerando este cenário, o presente artigo apresenta uma proposta para apoiar a acessibilidade de PCDVs (Pessoas com Deficiência Visual) total ou parcial, tendo como objetivo central contribuir com a inclusão social destas pessoas, promovendo uma maior independência e aumento da qualidade de vida das mesmas.

Para tanto, é concebido o projeto denominado HELIX, o qual integra recursos de hardware, firmware e software, explorando a ciência de contexto na UbiComp, na perspectiva de uma infraestrutura da Internet das Coisas. Nesse sentido, a abordagem proposta para o HELIX está integrada ao Subsistema de Adaptação e Reconhecimento de Contexto do middleware EXEHDA [Lopes et al. 2014]. O EXEHDA, enquanto middleware para UbiComp, provê suporte para a aquisição, armazenamento e processamento das informações de contexto necessárias às diferentes funcionalidades providas pelo HELIX.

O artigo está estruturado da seguinte forma: a seção 2 apresenta as funcionalidades do HELIX, seus aplicativos móveis, e o servidor de contexto. Os trabalhos relacionados são apresentados na seção 3. A seção 4 destaca a prototipação e a avaliação realizada. Por fim, na seção 5 são feitas as considerações finais deste trabalho.

\section{Funcionalidades do HELIX}

A abordagem proposta para o HELIX considera as premissas da área de tecnologia assistiva, sendo sua questão central atender as necessidades dos usuários tendo como pressupostos potencializar a autonomia e minimizar esforços de configuração, promovendo uma operação o mais transparente possível para as PCDVs. Para tanto, a abordagem explora a sinergia da ciência de contexto na UbiComp com recursos da computação móvel, disponibilizando de uma infraestrutura de Internet das Coisas, onde os objetos inteligentes da IoT podem interoperar.

$\mathrm{Na}$ IoT o relacionamento entre os objetos pode acontecer sem a intervenção humana. Deste modo, um objeto com funcionalidades de servidor pode ser responsável por descobrir situações em outros objetos, e articular relações, empregando mecanismos de ciência de contexto [Leal et al. 2013].

$\mathrm{Na}$ Internet das Coisas considera-se que devem ser mantidos separados os níveis de "pessoas" e "coisas", permitindo que objetos tenham suas próprias interações e os seres humanos especifiquem regras tanto para proteger sua privacidade, como para acessar os resultados das interações autônomas entre os objetos [Atzori et al. 2012].

Considerando as premissas da IoT e os objetivos do trabalho, foram concebidas as funcionalidades a serem contempladas no projeto HELIX. Uma visão geral destas funcionalidades é apresentada na Figura 1, tendo seus atores as seguintes características:

- Cuidador Pessoal: indivíduo responsável por prover auxílio à PCDV, pode ser um indivíduo familiar ou até mesmo um profissional contratado que fique disponível para atender as diferentes notificações do HELIX referente à PCDV. Uma mesma 
PCDV poderá ter diferentes cuidadores pessoais cadastrados, os quais serão acionados em sequência, cuja ordem deverá ser previamente definida. Uma vez atendida a notificação por um dos cuidadores, a sequência de notificações será interrompida.

- Cuidador Corporativo: indivíduo responsável pela zeladoria, auxílio e segurança de pessoas em ambientes corporativos. Também, atende às notificações do HELIX referentes às PCDVs na sua área de cobertura empresarial.

- PCDV Indoor: pessoa com deficiência visual ativa em ambientes interno, a qual irá realizar a leitura de QR-Codes como mecanismo de localização. Alguns QR-Codes localizados em posições estratégicas, de conteúdo fixo ou dinâmico (provido por Totens), também poderão ser empregados para disponibilização de informações especializadas.

- PCDV Outdoor: pessoa com deficiência visual atuando em ambientes externos, para a qual o HELIX irá, em períodos de tempo previamente cadastrados, informar automaticamente a localização da PCDV.

- Servidor do HELIX: executa as atividades de reconhecimento de contexto e provê suporte à infraestrutura da IoT.

As PCDVs e seus cuidadores deverão utilizar um smartphone com recurso de GPS (Global Positioning System) no qual os aplicativos móveis do HELIX serão instalados.

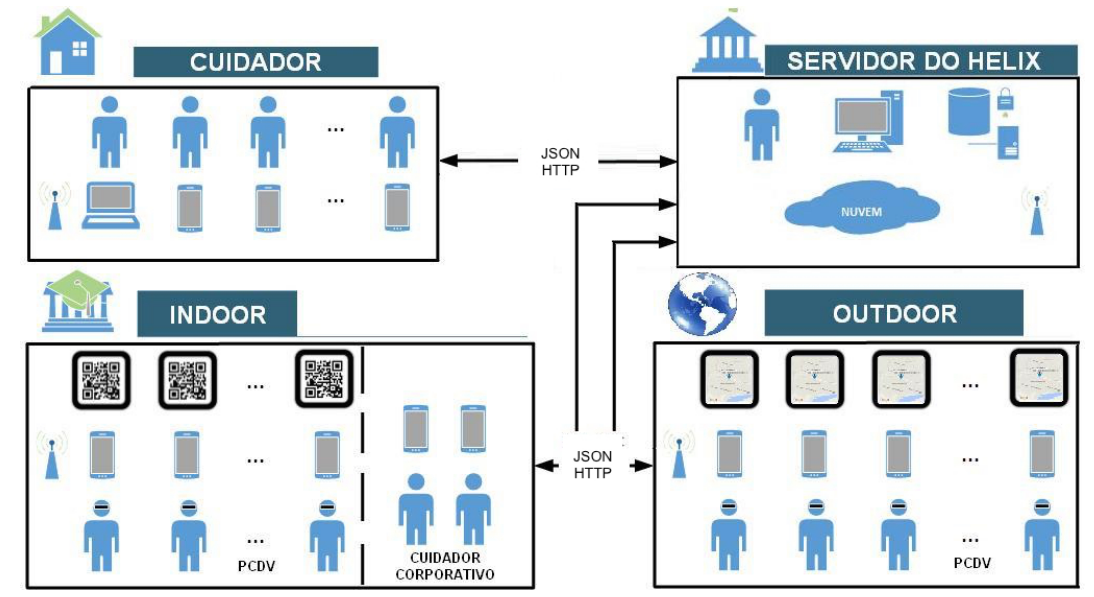

Figura 1. HELIX: Visão Geral das Funcionalidades

As funcionalidades do HELIX exploram os recursos do Subsistema de Reconhecimento de Contexto a Adaptação do middleware EXEHDA (vide Figura 2). Este subsistema é responsável pela coleta, armazenamento e processamento dos dados de contexto, provendo ciência de contexto. Para tanto, possui dois tipos de servidores: (i) Servidor de Borda: responsável por interagir com ambiente através de sensores e atuadores, realizando a coleta dos dados de contexto, bem como a execução de regras de contingência (prioritárias) e o armazenamento temporário das informações contextuais coletadas, em caso de falha de comunicação. (ii) Servidor de Contexto: responsável por prover o armazenamento e o processamento dos dados contextuais, integrando informações históricas e aquelas provenientes de diferentes Servidores de Borda distribuídos no ambiente ubíquo. 


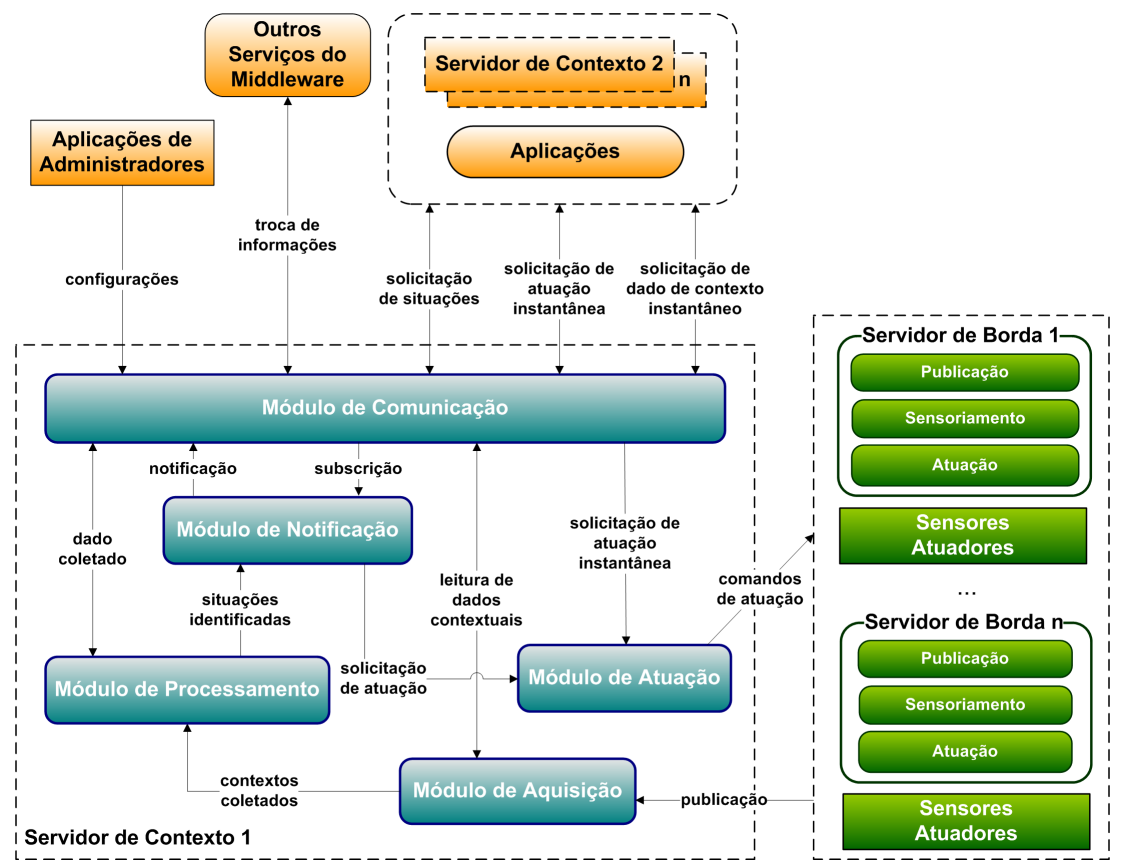

Figura 2. Subsistema de Reconhecimento de Contexto e Adaptação do EXEHDA

Estes servidores são alocados em células do ambiente ubíquo gerenciado pelo EXEHDA, onde cada célula possui um único Servidor de Contexto e pode possuir vários Servidores de Borda (vide Figura 3).

Considerando a infraestrutura de servidores do EXEHDA, descrita acima, os Aplicativos Móveis (Assistente e Monitores) do HELIX atuam como Servidores de Borda. Já as funcionalidades de provimento de ciência do contexto, típicas do Servidor de Contexto, são contempladas pelo Servidor do HELIX.

Na continuidade são caracterizadas as funcionalidades dos Aplicativos Móveis e do Servidor, empregados pelos atores do HELIX.

\section{Assistente Móvel de Acessibilidade (AMA)}

O AMA é o aplicativo instalado no smartphone que fica de posse da PCDV. Ele é responsável por prestar suporte diretamente na obtenção das informações contextuais relevantes a localização do usuário e também prestar auxílio, caso o usuário se encontre perdido ou tenha alguma eventual emergência.

Como o AMA é utilizado por PCDVs, não se pode utilizar a tela como forma de retorno sobre as ações executadas. Porém, através da memorização, pessoas com experiência podem ativar funcionalidades de aplicativos com tela sensível ao toque sem a necessidade de olhar para o dispositivo [Becker et al. 2009].

Apesar da possibilidade da memorização dos recursos oferecidos por uma interface, com o intuito de qualificar a relação com o seu usuário PCDV, o AMA utiliza um processo de leitura audível de sua interface. Essa leitura acontece sempre que o usuário manter o dedo pressionado sobre um dos botões que compõem a sua interface por alguns segundos, assim, o usuário terá a certeza de qual funcionalidade está selecionando sem executar o comando associado. 


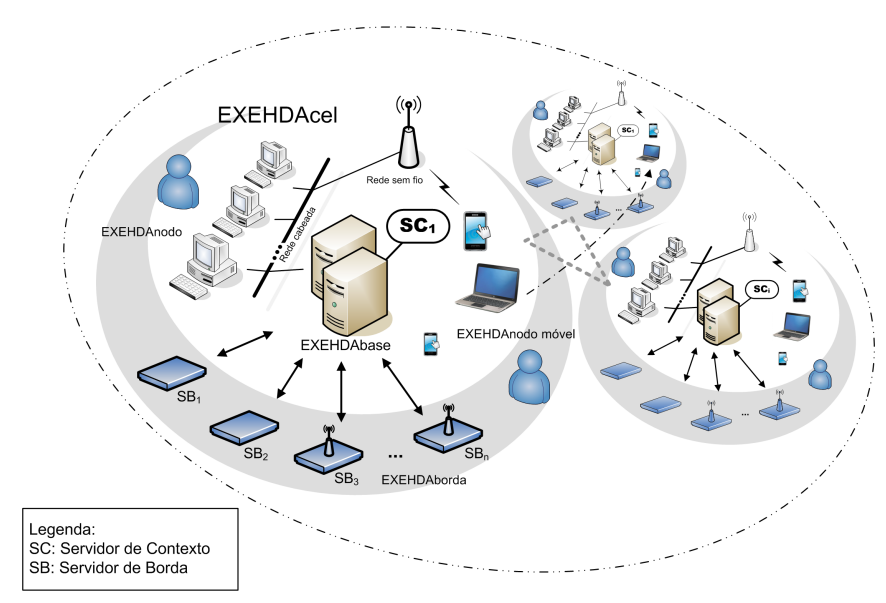

Figura 3. Ambiente Ubíquo Gerenciado pelo EXEHDA

São duas as possibilidades de localização da PCDV, a primeira delas acontece Indoor, sendo baseada na leitura de QR-Codes localizados em portas, elevadores, totens, balcões de atendimento, etc. Além de transmitir ao deficiente a informação sobre a sala ou ambiente em que ele se encontra, o aplicativo também envia a localização da PCDV ao Servidor do HELIX.

A outra possibilidade de localização ocorre Outdoor, na qual é utilizado o mecanismo de GPS do smartphone para auxiliar tanto a PCDV na sua rota como também na busca de locais previamente cadastrados como pontos de apoio à PCDVs (farmácias, postos de gasolina, lojas, etc.). Além disso, o aplicativo informa periodicamente ao Servidor do HELIX a localização do deficiente, tornando possível gerar alertas de segurança à PCDV e ao seu cuidador toda vez que a mesma se afastar de pontos de referência previamente cadastrados.

O retorno audível empregado pelo AMA utiliza a mesma linguagem padrão selecionada nas configurações de idioma do smartphone, portanto, se o usuário trocar a linguagem nativa do seu smartphone, a linguagem audível do AMA também mudará automaticamente. Essa característica potencializa a internacionalidade da solução.

O AMA também dispõe de um botão de emergência (panic button), o qual uma vez acionado envia ao Servidor do HELIX uma solicitação de ajuda associada a posição atual do PCDV capturada do GPS.

\section{Monitor Móvel de Acessibilidade (MMA)}

O MMA é o aplicativo instalado no smartphone que fica de posse pelo responsável da PCDV, sendo facultado que um deficiente visual possa ter mais de um responsável.

O objetivo do MMA é informar ao responsável pela pessoa deficiente todos os eventos que acontecem no AMA da PCDV, assim, promovendo um acompanhamento da mesma.

Esses eventos chegam ao usuário do MMA por meio de notificações enviadas pelo Servidor do HELIX. O responsável pela PCDV terá disponível, desde que previamente acordado, um histórico das Etiquetas de Acessibilidade lidas, contendo as informações de local, data e horário em que a PCDV realizou a captura da mesma. 
Quando ocorre a notificação de uma emergência pela PCDV, o usuário do MMA receberá junto com a solicitação de ajuda, uma imagem contendo a posição via GPS da PCDV.

\section{Monitor Móvel Empresarial (MME)}

O MME é um aplicativo corporativo instalado no smartphone de posse de um responsável por prestar suporte in-loco aos usuários dos AMAs dentro da área de cobertura do HELIX, que necessitem de ajuda, por exemplo, universidades, metrôs, estádios, aeroportos, etc.

Ao receber a solicitação de ajuda, o MME executa uma rotina de alerta visual e sonoro, no qual, ao ser confirmado pelo seu usuário, mostrará a localização e as informações de nome e número do telefone da PCDV que necessita de ajuda. Assim, o usuário do MME poderá ligar para a PCDV e verificar a sua necessidade ou ainda ir até a localização da mesma para atendê-la da forma mais rápida e eficiente possível.

\section{Servidor do HELIX}

O Servidor do HELIX utiliza a infraestrutura de software do Servidor de Contexto do middleware EXEHDA, particularmente emprega a estratégia híbrida para processamento das informações contextuais do Módulo de Processamento, a qual combina regras do tipo ECA (Evento-Condição-Ação) com a técnica de árvore de decisão [Han and Kamber 2006] para determinar as prioridades de atendimento das solicitações dos Aplicativos Móveis, tanto na operação indoor quanto na operação outdoor, a fim de gerenciar os alertas e a busca pelo cuidador mais próximo, bem como pontos de interesse e apoio previamente cadastrados.

Sendo assim, as alternativas de cuidadores ganham pesos diferentes, bem como suas probabilidades de atendimento. A decisão pode basear-se em estimativas e probabilidades associadas aos resultados de ações correntes que competem entre si. $\mathrm{O}$ resultado de cada solicitação é ponderado pela probabilidade associada a ela e a ação é então determinada de acordo com a melhor resposta.

Essencialmente, árvores de decisões são utilizadas para resolver problemas que envolvem decisões sequenciais, colocando em destaque os riscos e os resultados identificados nos diversos cursos das ações, caracterizando assim uma das melhores técnicas para aplicações de Internet das Coisas [Atzori et al. 2012].

\section{Trabalhos Relacionados}

Nesta seção são apresentados alguns dos principais trabalhos relacionados estudados. O critério de seleção foi sua proximidade com os objetivos perseguidos pelo HELIX, no que tange a acessibilidade para Pessoas com Deficiência Visual - PCDVs, utilizando tecnologias assistivas de UbiComp.

O PocketNavigator [Pielot et al. 2010] é um aplicativo para smartphone desenvolvido no Institute for Information Technology Oldenburg, na Alemanha. O aplicativo do projeto se propõe a orientar os seus usuários por meio de diferentes padrões de vibração do aparelho utilizado em ambientes outdoor, afim de informar o caminho a ser seguido, até o local de destino pré-selecionado. Assim, o usuário pode dispensar a necessidade de interação constante com a tela do dispositivo, podendo até mesmo deixá-lo 
no bolso. O aplicativo utiliza a estrutura de mapas do Google para a navegação. É possível utilizar a navegação mesmo sem rede, porém é necessário fazer o download do mapa da região desejada.

O modelo Tirésias [Falk et al. 2013] foi desenvolvido no Programa de Pós-Graduação em Computação Aplicada da Universidade do Vale do Rio dos Sinos (UNISINOS). O Tirésias é baseado no modelo Hefestos, o qual propõe um modelo de suporte à acessibilidade que utiliza a UbiComp para o gerenciamento de recursos indoor e outdoor. Com isso, enfoca diversos padrões de acessibilidade, não sendo orientado a um tipo específico de deficiência, apesar de ter sido validado com pessoas com deficiência motora. O Tirésias pode ser compreendido como uma especialização do Hefestos voltado especificamente para PCDV. O Tirésias é constituído por três módulos (entrada, saída e configuração) e um assistente pessoal (APP). O projeto considerou recursos reais de mapeamentos no campus da UNISINOS, onde pontos considerados relevantes para as PCDV foram mapeados por coordenadas GPS. O aplicativo torna possível o usuário escolher o seu destino em uma lista de opções pré-definidas e após o usuário recebe o suporte com informações relevantes por meio audível.

O SmartVision [Teixeira 2010] é um sistema de navegação e orientação para cegos que foi desenvolvido no campus da UTAD - Universidade de Trás-os-Montes e Alto Douro. Foi idealizado para funcionar em ambientes indoor e outdoor, recorrendo a diversas tecnologias de apoio. Dentre estas tecnologias, destaca-se a localização híbrida que permite aferir a posição do usuário em interiores e exteriores de edifícios de modo redundante, assegurando a localização a qualquer momento. A proposta da solução SmartVision utiliza para a navegação de PCDVs em ambiente outdoor tecnologias GPS e em ambientes indoor utiliza-se de etiquetas com tecnologia RFID - (Radio Frequency Identification) e redes WI-FI.

A Tabela 1 resume a análise dos trabalhos relacionados, incluindo o modelo proposto. Tanto o HELIX, como o Tirésias não consideram um hardware específico, pois foram desenvolvidos como aplicação para smartphones disponíveis no mercado.

O HELIX é o único modelo que utiliza etiquetas QR-Code, a qual pode ser lida por qualquer smartphone com câmera integrada, o que não acontece com as demais propostas que necessitam de leitores específicos de RFID. Do mesmo modo, o HELIX é a única proposta que se utiliza de uma infraestrutura da IoT para prover informações às PCDVs e aos seus cuidadores.

Ainda, tanto o HELIX como o Tirésias permitem uma fácil configuração das suas funcionalidades, sendo considerado esse aspecto crítico para a qualidade do sistema assistivo.

\section{Prototipação e Avaliação do HELIX}

No desenvolvimento das aplicações prototipadas para avaliação das funcionalidades do HELIX foram empregadas as seguintes tecnologias:

- Smartphones Android, nos quais foram desenvolvidos os Aplicativos Móveis.

- Ferramenta Android Studio para desenvolvimento do software dos Aplicativos Móveis. 
Tabela 1. Comparação entre os trabalhos relacionados.

\begin{tabular}{|c|c|c|c|}
\hline Proposta & $\begin{array}{l}\text { Hardware } \\
\text { Mercado }\end{array}$ & QR-Code & IoT \\
\hline PocketNavigator & Não & Não & Não \\
\hline Tirésias & Sim & Não & Não \\
\hline SmartVision & Não & Não & Não \\
\hline HELIX & Sim & Sim & Sim \\
\hline
\end{tabular}

- Padrão arquitetural REST [Fielding 2000] para a comunicação entre os Aplicativos Móveis e o Servidor do HELIX.

- Linguagem Python para programação das funcionalidades do Servidor do HELIX.

Na Figura 4 é possível visualizar algumas telas dos aplicativos do HELIX: a) tela do AMA; b) tela do MMA; e c) a tela do MME.

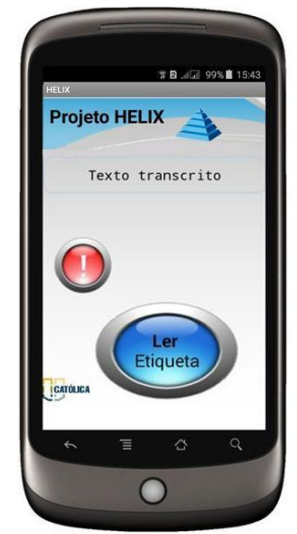

a)

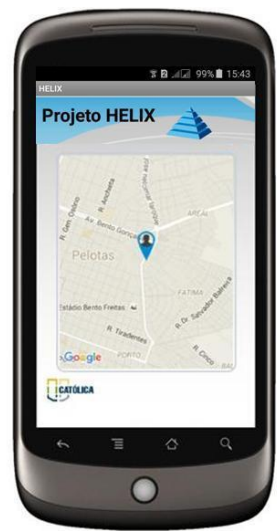

b)

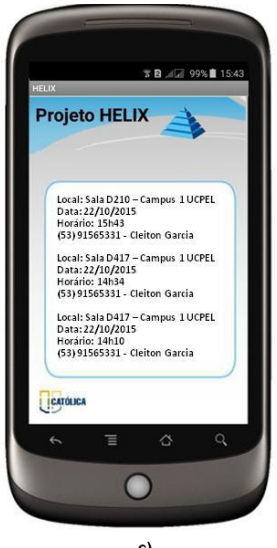

c)

Figura 4. Telas dos Aplicativos do HELIX

\subsection{Gerenciamento dos Recursos}

Atendendo a premissa de que as informações devem estar acessíveis independentemente do dispositivo computacional, as tecnologias utilizadas pelas aplicações no âmbito do projeto HELIX devem possuir recursos que permitam se ajustarem às mudanças ocorridas no ambiente de execução. Considerando isto, na prototipação da aplicação Web foram empregadas tecnologias responsivas no seu layout. O layout é considerado responsivo, quando ele se adapta ao tamanho da tela do dispositivo, sem prejudicar suas funcionalidades. Portanto, a aplicação pode ser acessada da mesma forma, tanto por desktops e notebooks como por tablets e smartphones.

A aplicação para gerência dos recursos de sensoriamento e atuação desenvolvida para o Servidor de Contexto é responsável por realizar as operações básicas utilizadas em banco de dados relacionais, ou seja, está capacitada a cadastrar, ler, atualizar e remover dados na camada de armazenamento do Repositório de Informações Contextuais. A aplicação foi desenvolvida utilizando princípios arquiteturais REST, possibilitando que qualquer operação possa ser realizada por uma URI específica. Ao acessar cada URI, parâmetros devem ser passados viabilizando assim que diversas funções requisitadas pelo Servidor de Contexto possam ser efetuadas. 
O acesso à aplicação de gerenciamento somente acontece por meio de login, onde o usuário poderá cadastrar os cuidadores da PCDV e a ordem de prioridade de atendimento de alertas conforme o contexto (local, horário, etc.).

Considerando estes aspectos, algumas funcionalidades foram criadas, as quais estão descritas a seguir:

- Servidores de borda: permite cadastrar todos os atores do HELIX.

- Regras: permite que sejam carregadas regras em arquivos Python, vinculadas aos assistentes móveis e/ou contextos de interesse para serem utilizadas no processamento das informações contextuais.

- Sensores: responsável por configurar os sensores (botões dos componentes), bem como a vinculação de cada sensor ao componente o qual estará atrelado.

A aplicação no seu modo de visualização possibilita a seleção de um ou mais sensores, dentro de um contexto de interesse, para exibição do registro histórico das suas informações contextuais. Na Figura 5 pode-se verificar o histórico de leitura de QR-Codes por uma PCDV, estas informações são apresentadas na forma de um relatório textual.

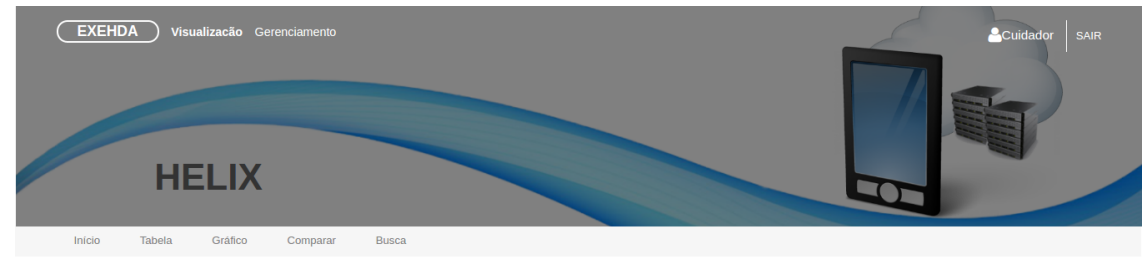

Sensor: QrCode

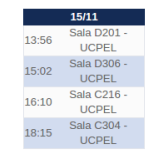

Figura 5. Tela de Visualização do Histórico das Leituras das Etiquetas QR-Codes de uma PCDV

\subsection{Avaliação de Usabilidade}

Para avaliação da usabilidade das aplicações prototipados para o HELIX foi utilizado o modelo TAM (Technology Acceptance Model) [Yoon and Kim 2007]. O TAM foi projetado para compreender a relação causal entre variáveis externas de aceitação dos usuários e o uso real de uma tecnologia da informação, buscando entender o comportamento deste usuário através do conhecimento da utilidade e da facilidade de uso percebida por ele. O TAM normalmente é utilizado para entender os motivos que levam os usuários a aceitar ou rejeitar uma tecnologia de informação e como melhorar a aceitação, oferecendo, desse modo, um suporte para prever e explicar a aceitação.

Nesta avaliação, após apresentação e exploração das funcionalidades do protótipo do HELIX em um ambiente controlado por professores e cuidadores da Escola Especial Louis Braille da cidade de Pelotas - RS, foi aplicado o formulário correspondente ao TAM, 
considerando os seguintes níveis de afirmação da escala Likert: Discordo plenamente; Discordo parcialmente; Indiferente; Concordo parcialmente; Concordo plenamente.

Com base em reuniões com o grupo de pesquisa e em comum acordo com professores e cuidadores da Escola Louis Braile, o formulário foi elaborado com as seguintes afirmações:

1. Os Aplicativos Móveis do Helix são fáceis de utilizar.

2. Os recursos do HELIX são úteis para prover acessibilidade às PCDVs.

3. O "Panic Button" é um importante diferencial do HELIX para prover segurança para as PCDVs.

4. O envio da mensagem com o estado da bateria com baixa autonomia do Smartphone da PCDV para o cuidador é relevante.

5. O histórico de localização das PCDVs e o envio de alertas é uma funcionalidade efetivamente útil para os cuidadores.

6. O uso do HELIX é recomendado para PCDVs.

7. Deve ser estabelecida uma lista com prioridades de cuidadores para o atendimento de chamadas da PCDV.

8. A responsabilidade de supervisionar a PCDV deve ser repassada automaticamente para o próximo cuidador da lista quando o cuidador principal não estiver online.

9. É realmente uma vantagem utilizar somente o Smartphone e não necessitar de um hardware adicional.

10. O fato do HELIX necessitar de conexão com Internet compromete o seu uso na prática.

11. O procedimento de leitura de QR Codes é viável por PDCVs.

Os resultados obtidos com a aplicação do formulário construído com o modelo TAM foram tabulados em planilha eletrônica e encontram-se disponíveis na Figura 6.

Na Figura 7 pode-se observar de forma simplificada a aceitação do grupo participante da pesquisa. Em barra hachurada verde o percentual de respondentes que concordaram plenamente com as afirmações do formulário aplicado.

As afirmações 9 e 10 foram as que chamaram a atenção pela maior dispersão das opiniões. Em relação à afirmação 9 sabe-se que o HELIX não é uma arquitetura que no momento resolva todas as necessidades de acessibilidade de PCDVs e este foi o maior motivo pelo qual alguns participantes da pesquisa discordaram do aspecto de se usar somente o smartphone como tecnologia assistiva. Algumas PCDVs possuem outros dispositivos que auxiliam na suas atividades do dia a dia como, por exemplo, um colorímetro.

No que tange à afirmação sobra a conexão com Internet, alguns participantes da pesquisa realizaram comentários escritos nos formulários a respeito do assunto, e ficou explícito que o problema do uso da tecnologia esbarra em questões financeiras da amostra populacional que respondeu ao formulário TAM.

Não foi foco do TAM aplicado estratificar os respondentes, pois a intenção era conhecer no seu nível mais abrangente possível as opiniões de pessoas ligadas à comunidade de PCDV. 


\begin{tabular}{|c|c|c|c|c|c|c|c|c|c|c|c|c|}
\hline \multirow[b]{2}{*}{ Participantes } & \multicolumn{11}{|c|}{ Questôes } & \multirow[b]{2}{*}{$\Sigma$} \\
\hline & 1 & 2 & 3 & 4 & 5 & 6 & 7 & 8 & 9 & 10 & 11 & \\
\hline 1 & 5 & 5 & 2 & 5 & 5 & 5 & 5 & 5 & 2 & 1 & 5 & 45 \\
\hline 2 & 5 & 5 & 5 & 5 & 5 & 5 & 5 & 5 & 4 & 5 & 5 & 54 \\
\hline 3 & 5 & 5 & 5 & 5 & 5 & 5 & 5 & 5 & 5 & 5 & 5 & 55 \\
\hline 4 & 5 & 5 & 5 & 5 & 5 & 5 & 5 & 5 & 5 & 4 & 5 & 54 \\
\hline 5 & 5 & 5 & 5 & 5 & 5 & 5 & 5 & 5 & 5 & 4 & 5 & 54 \\
\hline 6 & 5 & 5 & 5 & 5 & 5 & 5 & 5 & 5 & 5 & 4 & 4 & 53 \\
\hline 7 & 4 & 5 & 5 & 5 & 5 & 5 & 5 & 5 & 5 & 5 & 5 & 54 \\
\hline 8 & 5 & 5 & 5 & 5 & 5 & 5 & 5 & 5 & 5 & 5 & 5 & 55 \\
\hline 9 & 5 & 5 & 5 & 5 & 5 & 5 & 5 & 5 & 5 & 5 & 5 & 55 \\
\hline 10 & 5 & 5 & 5 & 5 & 5 & 5 & 5 & 5 & 5 & 1 & 5 & 51 \\
\hline 11 & 5 & 5 & 5 & 5 & 5 & 4 & 4 & 5 & 5 & 5 & 5 & 53 \\
\hline 12 & 5 & 5 & 5 & 5 & 5 & 5 & 5 & 5 & 3 & 5 & 5 & 53 \\
\hline 13 & 4 & 5 & 5 & 5 & 5 & 5 & 5 & 5 & 3 & 1 & 5 & 48 \\
\hline 14 & 3 & 5 & 5 & 5 & 5 & 5 & 5 & 5 & 2 & 1 & 5 & 46 \\
\hline 15 & 1 & 5 & 4 & 5 & 5 & 5 & 5 & 5 & 4 & 1 & 5 & 45 \\
\hline 16 & 5 & 5 & 5 & 5 & 5 & 5 & 5 & 4 & 4 & 5 & 5 & 53 \\
\hline 17 & 5 & 5 & 5 & 4 & 5 & 5 & 4 & 5 & 4 & 1 & 5 & 48 \\
\hline 18 & 5 & 5 & 5 & 5 & 5 & 5 & 5 & 5 & 5 & 5 & 5 & 55 \\
\hline 19 & 5 & 5 & 5 & 5 & 5 & 5 & 5 & 5 & 5 & 4 & 5 & 54 \\
\hline 20 & 5 & 5 & 5 & 5 & 5 & 5 & 5 & 5 & 4 & 5 & 5 & 54 \\
\hline 21 & 5 & 5 & 5 & 5 & 5 & 5 & 5 & 5 & 5 & 4 & 5 & 54 \\
\hline 22 & 5 & 5 & 5 & 5 & 5 & 5 & 5 & 5 & 5 & 2 & 5 & 52 \\
\hline 23 & 5 & 5 & 5 & 5 & 5 & 5 & 5 & 5 & 5 & 3 & 5 & 53 \\
\hline 24 & 5 & 5 & 5 & 5 & 5 & 5 & 5 & 5 & 5 & 5 & 5 & 55 \\
\hline 25 & 5 & 5 & 5 & 5 & 5 & 5 & 5 & 5 & 5 & 5 & 5 & 55 \\
\hline 26 & 5 & 5 & 5 & 5 & 5 & 5 & 5 & 5 & 5 & 2 & 4 & 51 \\
\hline 27 & 4 & 5 & 5 & 5 & 5 & 5 & 5 & 5 & 4 & 5 & 5 & 53 \\
\hline Variância & 0,74 & 0,00 & 0,35 & 0,04 & 0,00 & $0,04 \mid$ & 0,07 & 0,04 & 0,83 & 2,68 & 0,07 & 9,394 \\
\hline
\end{tabular}

Figura 6. Resultado do TAM Aplicado aos Professores e Cuidadores na Escola Louis Braille

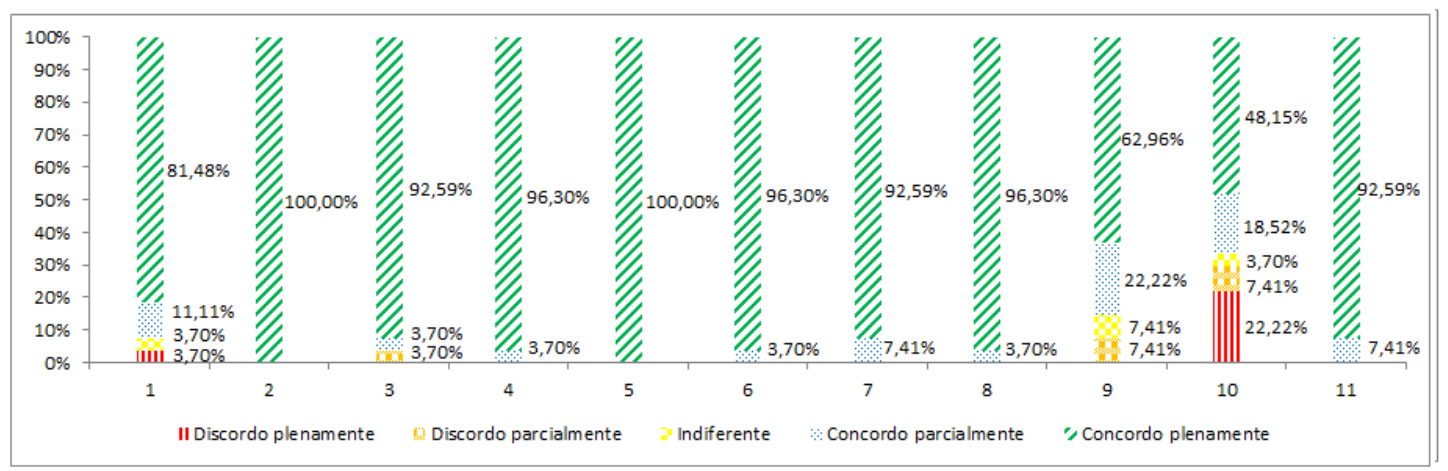

Figura 7. Gráfico de Barras Representando o TAM Aplicado aos Professores e Cuidadores na Escola Louis Braille

\section{Considerações Finais}

$\mathrm{O}$ trabalho desenvolvido apontou para a viabilidade de prover acessibilidade às pessoas com deficiência visual. A perspectiva buscada é potencializar a inclusão social de PCDVs, por meio de uma abordagem baseada na Internet das Coisas.

Nos trabalhos relacionados foi possível observar que existe uma preocupação de prover acessibilidade para pessoas com algum tipo de deficiência, utilizando a Computação Ubíqua. Por sua vez, o HELIX diferencia-se dos trabalhos estudados por empregar conceitos modernos como a IoT, explorando o atendimento através de uma rede distribuída de atores e componentes.

Como trabalhos futuros, identifica-se as seguintes frentes de atividades: 
(i) Submeter o HELIX a um Comitê de Ética a fim de realizar testes com humanos e assim poder caracterizar uma pesquisa clínica; (ii) Revisar a concepção dos Aplicativos Móveis para que se tornem compatíveis com os diversos modelos e versões de dispositivos móveis; (iii) Incluir um retorno audível após o usuário do AMA pressionar o botão de "Panic Button", para que o mesmo seja informado que sua solicitação de emergência chegou ao seu responsável; (iv) Trabalhar na escalabilidade do projeto, permitindo que o mesmo possa se comunicar com a central de atendimento, provendo mais uma característica de IoT às PCDVs; e (v) Revisar os aspectos de segurança do HELIX com a perspectiva de garantir a privacidade dos usuários.

\section{Referências Bibliográficas}

Atzori, L., Iera, A., Morabito, G., and Nitti, M. (2012). The social internet of things (siot)-when social networks meet the internet of things: Concept, architecture and network characterization. Computer Networks, 56(16):3594-3608.

Becker, E., Le, Z., Park, K., Lin, Y., and Makedon, F. (2009). Event based experiments in an assistive environment using wireless sensor networks and voice recognition. In Proceedings of the 2nd International Conference on PErvasive Technologies Related to Assistive Environments, page 17. ACM.

Falk, R. A., Tavares, J. E. d. R., and Barbosa, J. L. V. (2013). Tirésias: um modelo para acessibilidade ubíqua orientado à deficiência visual. Revista Brasileira de Computação Aplicada, 5(1):55-70.

Fielding, R. T. (2000). Architectural Styles and the Design of Network-based Software Architectures. Phd thesis, University of California, California-USA.

Han, J. and Kamber, M. (2006). Data Mining : Concepts and Techniques.

Leal, A. G., Santos, A. S. d., Noda, M. K., and Rodrigues, L. C. d. S. (2013). Internet social das coisas como agente agregador nas cidades inteligentes no brasil e no mundo. III Congresso Internacional do Conhecimento e Inovação, CIKI, 2013, Porto Alegre., pages 974-986.

Lopes, J. L., de Souza, R. S., Geyer, C. F. R., da Costa, C. A., Barbosa, J. L., Pernas, A. M., and Yamin, A. C. (2014). A middleware architecture for dynamic adaptation in ubiquitous computing. J. UCS, 20(9):1327-1351.

Pielot, M., Poppinga, B., and Boll, S. (2010). Pocketnavigator: vibro-tactile waypoint navigation for everyday mobile devices. In Proceedings of the 12th international conference on Human computer interaction with mobile devices and services, pages 423-426. ACM.

Silva, J. M., Rosa, J. H., Barbosa, J. L., Barbosa, D. N., and Palazzo, L. A. (2010). Content distribution in trail-aware environments. Journal of the Brazilian Computer Society, 16(3):163-176.

Teixeira, V. M. M. (2010). Sistema de localização hibrido para apoio à navegação de cegos no Campus Utad. Dissertação de mestrado, Universidade de Trás-os-Montes e Alto Douro, Vila Real, Portugal.

Yoon, C. and Kim, S. (2007). Convenience and tam in a ubiquitous computing environment: The case of wireless lan. Electron. Commer. Rec. Appl., 6(1):102-112. 